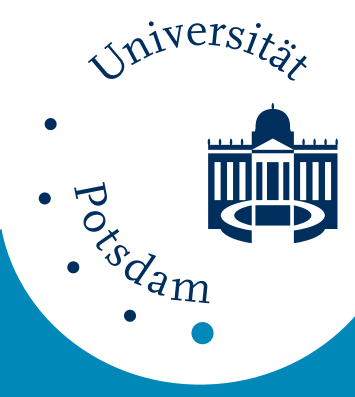

Universität Potsdam

Wolfgang Jansen

A note on the determination of the type of communication areas

NLD Preprints ; 33 


\title{
A Note on the Determination of the Type of Communication Areas
}

\author{
Wolfgang Jansen
}

Max-Planck-Gesellschaft zur Förderung der Wissenschaft AG Nichtlineare Dynamik an der Universität Potsdam Am Neuen Palais 10, Haus 19

PSF 601553

D-14415 Potsdam, Germany 


\begin{abstract}
The paper presents a method that determines, by standard numerical means, the type of mutual relations of fold and flip bifurcations (configured as a so-called communication area) of a map. Equation systems are developed for the computation of points where a transition between areas of different types occurs.

Furthermore, it is shown that saddle area $\leftrightarrow$ spring area transitions can exist which have not yet been considered in the literature. Analytical conditions of that transition are derived.
\end{abstract}

\title{
1 Introduction
}

The relations between fold and flip bifurcations of period- $k$ cycles of a map

$$
\boldsymbol{x}_{t+1}=\boldsymbol{f}\left(\boldsymbol{x}_{t}, \lambda\right)
$$

have been studied by Mira et al. in a series of papers (see [10], [11], [3], $[12],[13],[1],[2])$. In Eq. (1) $\boldsymbol{x}$ denotes the $n$-dimensional state vector, $\lambda$ is the $p$-dimensional parameter vector $(p \geq 3)$, and $\boldsymbol{f}$ is as smooth as needed (up to $C^{4}$ ). Mira [10] introduced so-called communication areas in the parameter plane. A communication area is a domain bordered by a (more or less arbitrary) smooth curve such that exactly two curves of fold bifurcations and two curves of flip bifurcations cross the area where all these curves correspond to cycles of the same period $k$. Furthermore, it is assumed that on one fold curve there is a cusp point and that all these bifurcation curves belong, in the state-parameter space, to a connected set of cycles. This means, that the communication area separates a specific bifurcation scenario from the possibly much more complex global bifurcation structure.

Three types of communication areas have been considered in the papers mentioned above: the saddle area $(S A A)$, the spring area $(S P A)$, and the crossroad area $(C R A)$. The different types of communication areas are distinguished by the position of the cusp point relatively to the other three curves. Their schemes are shown in Fig. 1 (more impressive three-dimensional drawings are presented in the papers mentioned). When a third parameter is varied the type of the communication area can change without changing the bifurcation structure on the area boundary: SPA $\leftrightarrow \mathrm{CRA}$ and SAA $\leftrightarrow \mathrm{CRA}$ transitions are known. 

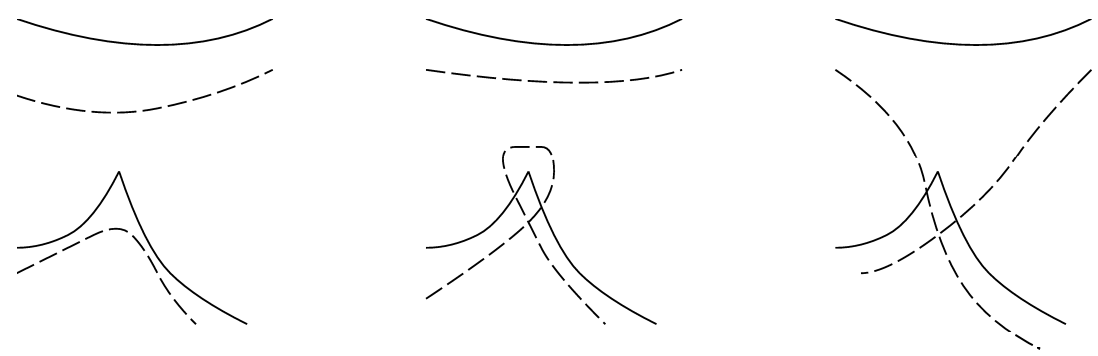

Figure 1: Scheme of the three communication areas: SAA (left), SPA (middle), CRA (right). Solid lines: fold bifurcations, dashed lines: flip bifurcations.

It is possible or even necessary that also other bifurcation curves are present in a communication area, e.g. often there exist flip bifurcation curves of the $2 k$-periodic cycle. Such additional bifurcation curves do not play any role in what follows, thus, we will neglect them. Furthermore, since all cycles considered for a given communication area have the same period $k$, we will always assume that $\boldsymbol{f}(\boldsymbol{x}, \lambda)$ is already the $k$-th iterate of the original map and, for simplicity of the presentation, we will never mention the actual period.

Carcassès $[1,2]$ presented a method that allows to determine the type of a communication area (in the 2-parameter plane) or to determine the point of an area transition (in 3-parameter space). The method is based on the so-called reduced multiplier $\sigma$, the solution of $\sigma \eta(1)+\theta(1)=0$ where $\eta(\mu)$ and $\theta(\mu)$ are the even and odd parts, respectively, of the characteristic polynomial $\chi(\mu)$ of the Jacobian $\boldsymbol{J}$ of $\boldsymbol{f}(\boldsymbol{x})$ computed at the cycle $\boldsymbol{x}$. When applying this method one has to solve nonlinear equation systems that contain equations of determinants which, in turn, have at least one row depending on derivatives of $\eta$ and $\theta$. This means, the first derivative of the characteristic polynomial is needed and, if Newton's method is used, even the second derivative.

The computation of the characteristic polynomial and of its derivatives is numerically difficult. The same has to be said about the solution of equation systems containing $n$-dimensional determinants. Thus, the general way to tackle bifurcation problems numerically is the construction of nonlinear equation systems which avoid determinants and the characteristic polynomial. The main goal of this paper is to show how the type of a communication area can be determined as a by-product of the computation of a cusp point by standard methods of the numerical bifurcation analysis. 
Moreover, we show that there is a new type of area transitions, the $\mathrm{SAA} \leftrightarrow \mathrm{SPA}$ transition. We present equation systems that determine those situations in the state-parameter space as well as equation systems that determine $\mathrm{SAA} \leftrightarrow \mathrm{CRA}$ transitions.

The paper is organized as follows. Section 2 gives a short review of definitions and results about the communication areas as well as the about numerical methods to compute a bifurcation point. After these preparations the formulas are derived in Sec. 3 that allow the determination of the type of a communication area and to compute area transitions, this is the main part of the paper. The last two sections provide the comparison with Carcassès' method (Sec. 4) and an example (Sec. 5), respectively.

\section{Preliminaries}

\subsection{Transitions of communication areas}

The SPA $\leftrightarrow$ CRA transition occurs when the flip curve surrounding the cusp and another flip curve (that separates the "spring" from the second fold curve) have contact to each other such that the connection of the four branches is reorganized and the way between the fold curves becomes free (see Fig. 2). This type of area transition is not local to the cusp and cannot be detected if the cusp is treated locally as we will do. A consequence is that we will not be able to distinguish between SPA and CRA. Instead, we will distinguish the type of a communication area by the alternative: SAA or not SAA.

The SAA $\leftrightarrow$ CRA transition is more complex from the geometrical point of view. The projection onto the two-parameter plane shows the following scenario. When a third parameter is varied the cusp point approaches the second fold curve such that, in the moment of contact, the branches of the cusp are tangent to that fold curve. After the contact, the cusp withdraws from the fold curve. But now the branches have exchanged: one branch of the cusp was, before the contact, a branch of the second fold curve and vice versa (see Fig. 2). The SAA $\leftrightarrow$ CRA transition is local to the cusp. Since it involves two curves where the Jacobians $\boldsymbol{J}$ have the eigenvalue +1 , this eigenvalue is degenerate, i.e. a specific third derivative of $\boldsymbol{f}(\boldsymbol{x}, \lambda)$ vanishes. Flip curves and the eigenvalue -1 do not play any local role but they are 

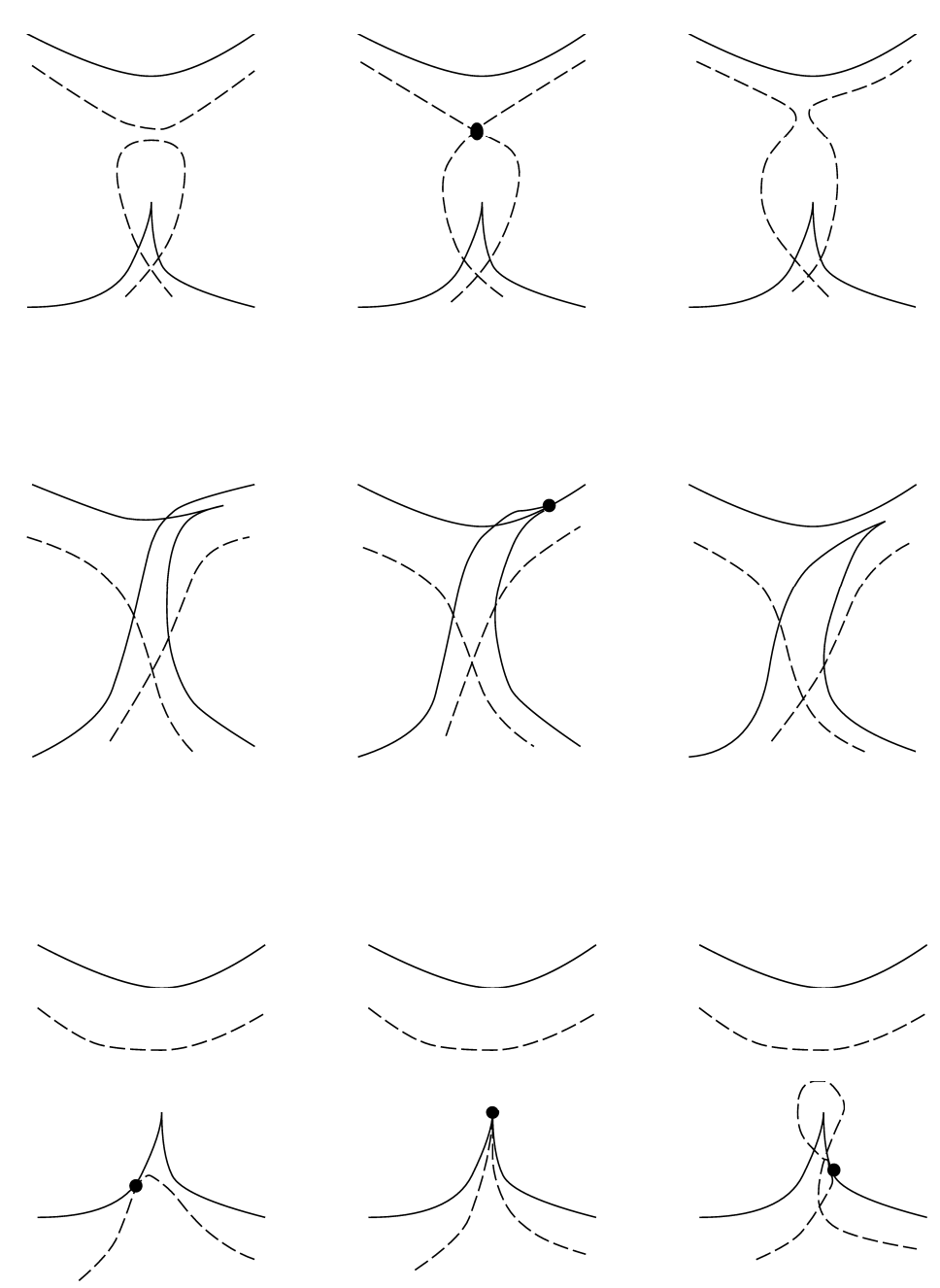

Figure 2: The area transitions: SPA $\leftrightarrow \mathrm{CRA}$ (top row), SAA $\leftrightarrow \mathrm{CRA}$ (middle row), SAA $\leftrightarrow$ SPA (bottom row). Solid lines: fold bifurcations, dashed lines: flip bifurcations, dots: the contact point of two curves. 
important from the global point of view. In fact, the reorganization of the fold branches causes that the positions of the flip curves relatively to the cusp are changed.

The SAA $\leftrightarrow$ CRA and SPA $\leftrightarrow$ CRA transitions involve only one type of codimension-2 bifurcations: the cusp. If also other co-dimension-2 bifurcations are admissible then further types of area transitions can occur. In particular, if a point of contact of a fold and a flip curve is taken into account then a SAA $\leftrightarrow$ SPA transition occurs when the contact point moves towards and eventually passes the cusp point (see Fig. 2). The SAA $\leftrightarrow$ SPA transition is local to the cusp. Immediately after the transition the communication area is a SPA, later it may become a CRA. On the defining fold and flip curves the Jacobians $\boldsymbol{J}$ have the eigenvalues +1 or -1 , respectively. This means, that $\boldsymbol{J}$ has both eigenvalues at the contact point, we will call such points \pm -bifurcations. In particular, the SAA $\leftrightarrow \mathrm{SPA}$ transition cannot occur in one-dimensional maps.

\subsection{Equation systems for bifurcation points}

Many equation systems have been developed for the computation of bifurcation points of different kind. We focus on equation systems of the following form

$$
\begin{aligned}
\boldsymbol{f}(\boldsymbol{x}, \lambda)-\boldsymbol{x} & =\boldsymbol{0} \\
\phi_{1}(\boldsymbol{x}, \lambda) & =0 \\
\vdots & \\
\phi_{m}(\boldsymbol{x}, \lambda) & =0
\end{aligned}
$$

The solution is a co-dimension- $m$ bifurcation and $m$ of the $p$ parameters are calculated (the others are held fixed). One or more of the functions $\phi_{1}, \ldots$, $\phi_{m}$ are obtained as the last component of the solution of the nonsingular linear equation systems

$$
\left(\begin{array}{cc}
\boldsymbol{M}_{i}(\boldsymbol{x}, \lambda) & \boldsymbol{r}_{i} \\
\boldsymbol{l}_{i}^{T} & 0
\end{array}\right)\left(\begin{array}{c}
\boldsymbol{u}_{i} \\
\phi_{i}
\end{array}\right)=\left(\begin{array}{l}
\boldsymbol{o} \\
1
\end{array}\right)
$$

with $i \in\{1, \ldots, m\}$. The $\boldsymbol{r}_{i}$ and $\boldsymbol{l}_{i}$ are fixed, appropriately chosen auxiliary vectors and the matrices $\boldsymbol{M}_{i}$ will be described later. Equation systems of 
this kind have been introduced by Griewank \& Reddien [6, 7] and Jepson \& Spence [9], an systematic approach to multiple eigenvalues gives Govaerts [5].

It is easy to see that $\phi_{i}=0$ iff $\operatorname{det} \boldsymbol{M}_{i}=0$. In this case, $\boldsymbol{u}_{i}$ is the (right) eigenvector to the zero eigenvalue and the matrix of Eq. (3) is nonsingular iff rank $M_{i}=n-1$ and $\boldsymbol{l}_{i}^{\prime} \boldsymbol{u}_{i} \neq 0$ as well as $\boldsymbol{v}_{i}^{T^{\prime}} \boldsymbol{r}_{i} \neq 0$. Here, $\boldsymbol{v}_{i}$ is the left eigenvector to the zero eigenvalue, i.e. the first part of the solution of

$$
\left(\begin{array}{ll}
\boldsymbol{v}_{i}^{T} & \phi_{i}
\end{array}\right)\left(\begin{array}{cc}
\boldsymbol{M}_{i}(\boldsymbol{x}, \lambda) & \boldsymbol{r}_{i} \\
\boldsymbol{l}_{i}^{T} & 0
\end{array}\right)=\left(\begin{array}{ll}
\boldsymbol{O}^{T} & 1
\end{array}\right) .
$$

At the bifurcation point, i.e. when $\operatorname{rank} M=n-1$, solving (3) can be understood as the transformation of the dynamical system to the center manifold: it is the numerical realization of the Lyapunov-Schmidt reduction.

For each eigenvalue specific for a bifurcation situation one has to provide one matrix $\boldsymbol{M}_{i}$ yielding a $\phi_{i}$ of Eq. (2). In our case, we have to set $\boldsymbol{M}_{i}=$ $\boldsymbol{J}-\boldsymbol{I}$ for fold points and $\boldsymbol{M}_{i}=\boldsymbol{J}+\boldsymbol{I}$ for flip points ( $\boldsymbol{I}$ denotes the unity matrix), both can occur simultaneously. But several of the functions $\phi_{1}$, $\ldots, \phi_{m}$ have to be constructed differently. We consider the situation of a cusp point where a degeneracy of a fold occurs. To be specific, let $m=2$ and let $\phi_{1}$ and $\phi_{2}$ describe the fold and the cusp conditions, respectively, i.e. $\phi_{1}$ is given by Eq. (3) and $\phi_{2}$ will be described next. If the map is restricted to the center manifold of a fold point then the second derivative of the map vanishes. Since the reduction to the center manifold is given by $z_{t+1}=z_{t}+\frac{1}{2 \boldsymbol{v}_{1}^{T} \boldsymbol{u}_{1}} \boldsymbol{v}_{1}^{T} \frac{\partial}{\partial \boldsymbol{x}} \boldsymbol{J} \boldsymbol{u}_{1} \boldsymbol{u}_{1} z_{t}^{2}+\cdots$, the cusp condition can be written as

$$
\phi_{2} \equiv-\boldsymbol{v}_{1}^{T}\left(\frac{\partial \boldsymbol{J}}{\partial \boldsymbol{x}} \boldsymbol{u}_{1}\right) \boldsymbol{u}_{1}=0
$$

Equation (2) with $m=2$ and these two functions is well posed iff the solution is a non-degenerate cusp point.

Our method to determine the type of a communication area is based on information that is gained by auxiliary calculations when computing a cusp point along a curve of fold points: a specific third derivative of $\boldsymbol{f}(\boldsymbol{x}, \lambda)$ is utilized. In the remainder of this section we will focus on that. Solving Eq. (2) by Newton's method (or a variant of it) needs the derivatives of the functions $\phi_{1}$ with respect to $\alpha$ ( $\alpha$ may be any component of $\boldsymbol{x}$ or $\lambda$ ). The 
equation $\frac{\partial \boldsymbol{A}^{-1}}{\partial \alpha}=-\boldsymbol{A}^{-1} \frac{\partial \boldsymbol{A}}{\partial \alpha} \boldsymbol{A}^{-1}$ for a nonsingular matrix $\boldsymbol{A}$ immediately yields

$$
\frac{\partial \phi_{1}}{\partial \alpha}=-\boldsymbol{v}_{1}^{T} \frac{\partial \boldsymbol{J}}{\partial \alpha} \boldsymbol{u}_{1}
$$

i.e. there is no need of solving further equation systems. Moreover, $\phi_{2}=$ $\frac{\partial \phi_{1}}{\partial \boldsymbol{x}} \boldsymbol{u}_{1}$, i.e. the value of the cusp condition is obtained as a by-product. The derivative of $\phi_{2}$ is given by

$$
\begin{aligned}
\frac{\partial \phi_{2}}{\partial \alpha}= & \boldsymbol{v}_{1}^{T} \frac{\partial \boldsymbol{J}}{\partial \alpha} \boldsymbol{A}\left(\frac{\partial \boldsymbol{J}}{\partial \boldsymbol{x}} \boldsymbol{u}_{1}\right) \boldsymbol{u}_{1} \\
& -\boldsymbol{v}_{1}^{T} \frac{\partial^{2} \boldsymbol{J}}{\partial \alpha \partial \boldsymbol{x}} \boldsymbol{u}_{1} \boldsymbol{u}_{1} \\
& +2 \boldsymbol{v}_{1}^{T}\left(\frac{\partial \boldsymbol{J}}{\partial \boldsymbol{x}} \boldsymbol{u}_{1}\right) \boldsymbol{A} \frac{\partial \boldsymbol{J}}{\partial \alpha} \boldsymbol{u}_{1}
\end{aligned}
$$

(here, $\boldsymbol{A}$ is the inverse matrix of Eq. (3) where the last row and last column have been deleted). This expression looks complicated but the effort to obtain it is proportional to $n$ and not to $n^{3}$ function calls since only the derivative of $\boldsymbol{J}$ in a definite direction is needed (a similar statement holds for all the second derivatives of $\boldsymbol{f}$ occurring somewhere in the algorithm). The cusp is non-degenerate iff

$$
\phi_{3} \equiv \frac{\partial \phi_{2}}{\partial \boldsymbol{x}} \boldsymbol{u}_{1}
$$

does not vanish, i.e. $\phi_{3}=0$ is the condition for a degenerate cusp point and can be included in Eq. (3) if such a is to be computed.

\section{Determination of the Type of a Communi- cation Area}

\subsection{The reduced multiplier}

Let $\chi(\mu)=\sum_{i=0}^{n} a_{n-i} \mu^{i}$ be the characteristic polynomial of the Jacobian matrix $\boldsymbol{J}$ computed at the cycle $\boldsymbol{x}$ and set $\chi_{+}=\chi(1), \chi_{-}=\chi(-1)$. Carcassès [2] defines the reduced multiplier $\sigma$ as the solution of

$$
\sigma \eta+\theta=0
$$


where

$$
\begin{aligned}
& \eta=a_{0}+a_{2}+\cdots= \begin{cases}\frac{1}{2}\left(\chi_{+}+\chi_{-}\right) & , n \text { even } \\
\frac{1}{2}\left(\chi_{+}-\chi_{-}\right) & , n \text { odd }\end{cases} \\
& \theta=a_{1}+a_{3}+\cdots= \begin{cases}\frac{1}{2}\left(\chi_{+}-\chi_{-}\right) & , n \text { even } \\
\frac{1}{2}\left(\chi_{+}+\chi_{-}\right) & , n \text { odd }\end{cases}
\end{aligned}
$$

i.e. $\sigma$ is the solution of

$$
(\sigma+1) \chi_{+}+(-1)^{n}(\sigma-1) \chi_{-}=0 .
$$

The equation has no unique solution iff $\chi_{+}=\chi_{-}=0$, in this case we let $\sigma$ undefined. Otherwise, the equation has no solution if $\chi_{+}+(-1)^{n} \chi_{-}=0$, in this case we set $\sigma=\infty$. It is easy to see that the reduced multiplier assumes the values +1 or -1 iff $\chi_{+}=0$ or $\chi_{-}=0$, i.e. iff the cycle belongs to a fold or a flip curve, respectively. The only exceptions are the \pm -bifurcations, these are the points where $\sigma$ is undefined.

To avoid the inconvenient situation $\sigma=\infty$ we consider together with $\sigma(\boldsymbol{x})$ the function $\hat{\sigma}(\boldsymbol{x})=\frac{1}{\sigma(\boldsymbol{x})}$. Exactly one of the functions $\sigma$ or $\hat{\sigma}$ takes on values in the interval $(-1,1)$ for cycles different from fold or flip points. We call $\sigma$ the even reduced multiplier and $\hat{\sigma}$ the odd one, furthermore, we call a cycle $\boldsymbol{x}$ such that the even or odd reduced multiplier is in the interval $(-1,1)$ an even or odd cycle, respectively.

By continuity of the functions $\sigma$ and $\hat{\sigma}$ the sets of even or odd cycles are open. This means, that any domain (i.e. any open, connected subset) of cycles not containing fold or flip points consists of even cycles only or of odd cycles only (otherwise, the domain were not connected). Accordingly, we call such a domain even or odd.

In the remainder of this section we will develop some analytic relations of the reduced multipliers. First of all, we express $\sigma$ by the determinants

$$
\begin{aligned}
& \Delta_{+}=\operatorname{det}\left(\begin{array}{cc}
\boldsymbol{J}-\boldsymbol{I} & \boldsymbol{r}_{+} \\
\boldsymbol{l}_{+}^{T} & 0
\end{array}\right), \\
& \Delta_{-}=\operatorname{det}\left(\begin{array}{cc}
\boldsymbol{J}+\boldsymbol{I} & \boldsymbol{r}_{-} \\
\boldsymbol{l}_{-}^{T} & 0
\end{array}\right) .
\end{aligned}
$$

Here and in the following, we index the matrix entries, determinants, and solutions of Eq. (3) not by "1" etc. (since their ordering is not important) 
but by "+" and "-" referring to the eigenvalue +1 or -1 to be computed. This is the same time the index of $\chi_{ \pm}$and the inverse of the sign of $\boldsymbol{I}$ in Eq. (3). Cramer's rule applied to Eq. (3) yields

$$
\begin{aligned}
& \chi_{+}=\operatorname{det}(\boldsymbol{J}-\boldsymbol{I})=\phi_{+} \Delta_{+}, \\
& \chi_{-}=\operatorname{det}(\boldsymbol{J}+\boldsymbol{I})=\phi_{-} \Delta_{-} .
\end{aligned}
$$

Let $\chi_{ \pm}$differentiably depend on a parameter $\alpha$. Then a straight forward computation shows that the derivative evaluated at a fold point $\mathcal{F}$ is simply

$$
\left.\frac{\partial \sigma}{\partial \alpha}\right|_{\mathcal{F}}=\left.(-1)^{n+1} \frac{2 \Delta_{+}}{\phi_{-} \Delta_{-}} \frac{\partial \phi_{+}}{\partial \alpha}\right|_{\mathcal{F}}
$$

(recall $\sigma-1=0$ and $\phi_{+}=0$ ). Moreover, in the case that also this derivative vanishes (i.e. at a cusp point $\mathcal{C}$ ) the second derivative is

$$
\left.\frac{\partial^{2} \sigma}{\partial \alpha^{2}}\right|_{\mathcal{C}}=\left.(-1)^{n+1} \frac{2 \Delta_{+}}{\phi_{-} \Delta_{-}} \frac{\partial^{2} \phi_{+}}{\partial \alpha^{2}}\right|_{\mathcal{C}} .
$$

\subsection{The concept}

In the remainder of the paper we suppose the following regularity properties (if not the contrary is stated explicitly): the type of the communication area in question is uniquely defined, i.e. it is not the situation of an area transition. In particular, $\boldsymbol{J}$ possesses at the cusp point $\mathcal{C}$ the eigenvalue +1 , no eigenvalue -1 , and $\mathcal{C}$ is non-degenerate. The condition on the eigenvalues implies $\chi_{+}=0$ and $\chi_{-} \neq 0$, i.e. both functions $\sigma$ and $\hat{\sigma}$ are defined and continuous in a neighborhood of $\mathcal{C}$. Similarly, when considering points $\mathcal{F}$ on the fold curve then they are to be neither cusp nor \pm -bifurcation points.

We will determine the type of a communication area by the sign of an arithmetic expression. More precisely, the product of two values is evaluated characterizing two specific domains of cycles which will be defined next.

The inner domain of a communication area is that sheet of the foliated set of cycles that is bounded by both branches of the cusp and that contains the acute angle of the cusp (see Fig. 3). A transient domain is a domain where the (even or odd) reduced multiplier varies from -1 to +1 , i.e. it is bordered by both fold and flip curves (see Fig. 3). In particular, the transient domain of a communication area is that containing the cusp point in its boundary. 

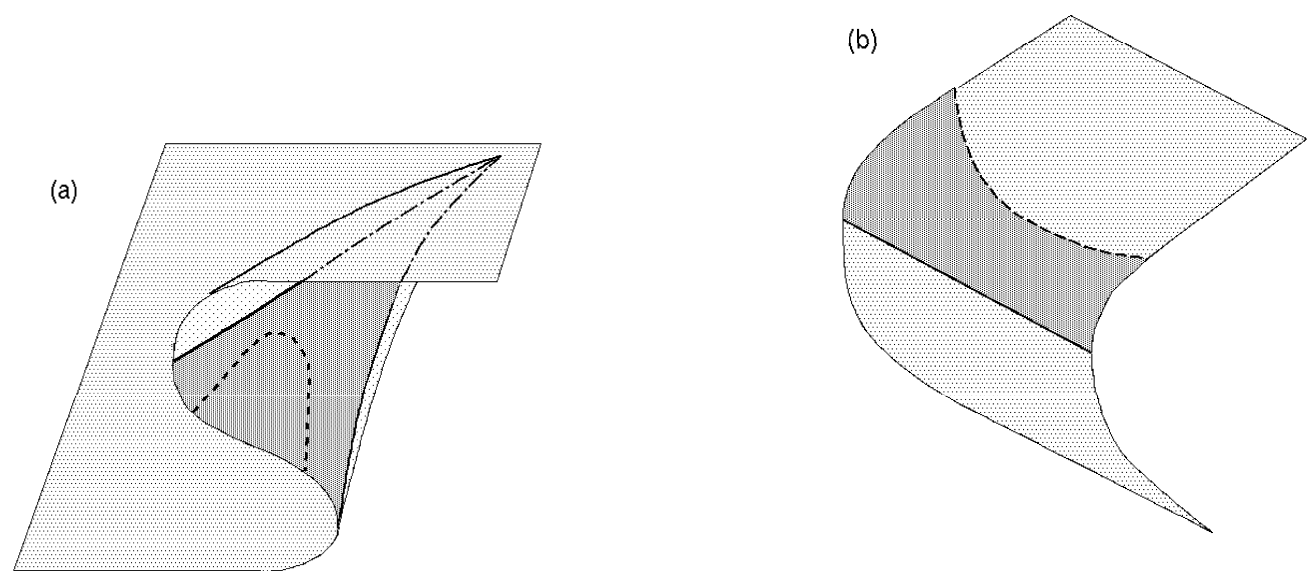

Figure 3: Specific domains (darkly shaded areas): (a) the transient domain between a fold and flip curve, (b) the inner domain of a cusp. Solid lines: fold curves, dashed lines: flip curves, dash-dotted line: hidden part of a fold curve.

This definition is correct since each communication area has exactly one such domain. We define $\epsilon_{\text {inner }}= \pm 1$ and $\epsilon_{\text {trans }}= \pm 1$ with " + " iff the corresponding domain is even. With other words, the domain is even iff $\epsilon_{\text {inner }}=(-1)^{m}$ or $\epsilon_{\text {trans }}=(-1)^{m}$ for an even number $m$ (this relation caused the terms "even" and "odd" domains).

Let $\mathcal{F}$ be a fold point. Applying Eq. (11) to the tangent $\boldsymbol{u}_{+}$at $\mathcal{F}$ yields

$$
\begin{aligned}
\left.\frac{\partial \sigma}{\partial \boldsymbol{x}}\right|_{\mathcal{F}} \boldsymbol{u}_{+} & =\left.(-1)^{n+1} \frac{2 \Delta_{+}}{\phi_{-} \Delta_{-}} \frac{\partial \phi_{+}}{\partial \boldsymbol{x}}\right|_{\mathcal{F}} \boldsymbol{u}_{+} \\
& =\left.(-1)^{n} \frac{2 \Delta_{+}}{\phi_{-} \Delta_{-}} \boldsymbol{v}_{+}^{T} \frac{\partial \boldsymbol{J}}{\partial \boldsymbol{x}}\right|_{\mathcal{F}} \boldsymbol{u}_{+} \boldsymbol{u}_{+} \neq 0
\end{aligned}
$$

by the cusp condition Eq. (5) since $\mathcal{F}$ is not a cusp point. This means, that on one side of the fold curve $\sigma$ takes on values $<1$ and values $>1$ on the other side, i.e. the domain on one side is even and that on the other side is odd. In particular, exactly one of the inner domain or its complement contains in a neighborhood of $\mathcal{C}$ an even domain.

The essential observation is the following. The communication area is a SAA iff its transient domain is a subset of the inner domain. Equivalently, it 
is a SAA iff both the inner and the transient domains are at the same time even or odd, i.e. iff $\epsilon_{\text {inner }} \epsilon_{\text {trans }}=1$ (see Fig 3: in case of a SAA the domain "between" the fold and the flip curves is part of the inner domain). Thus, our goal is to determine $\epsilon_{\text {inner }}$ and $\epsilon_{\text {trans }}$ near the cusp point.

\subsection{The transient domain}

Let $\Gamma_{1}$ be a smooth curve crossing a transient domain, let its end points at the fold and flip curves be $\mathcal{F}$ and $\mathcal{P}$, respectively. We assume that the curve is parameterized by $\alpha$ (increasing from $\mathcal{F}$ to $\mathcal{P}$ ). Furthermore, we can assume that $\Gamma_{1}$ is tangent to the vector $\boldsymbol{u}_{+}$at $\mathcal{F}$. Let $\epsilon_{u}=+1$ if $\boldsymbol{u}_{+}$points into that direction of $\Gamma_{1}$ where $\mathcal{P}$ lies, otherwise, let $\epsilon_{u}=-1$. The direction of $\boldsymbol{u}_{+}$and, consequently, the value of $\epsilon_{u}$ depend on the choice of the auxiliary vectors $\boldsymbol{r}_{+}$and $\boldsymbol{l}_{+}$. In the situation of Fig. 3 we have $\epsilon_{u}=1$.

If the cycle $\boldsymbol{x}$ is moved along $\Gamma$ from $\mathcal{F}$ in the direction of $\epsilon_{u} \boldsymbol{u}_{+}$, i.e. towards $\mathcal{P}$, then the reduced multiplier $\sigma$ decreases iff that transient domain is even, i.e. iff $\epsilon_{\text {trans }}=1$. On the other hand, $\sigma$ decreases iff its first nonvanishing derivative in the specified direction is negative. By Eq. (11),

$$
\begin{aligned}
\epsilon_{\text {trans }} & =-\left.\operatorname{sign} \frac{\partial \sigma}{\partial \boldsymbol{x}}\right|_{\mathcal{F}}\left(\epsilon_{u} \boldsymbol{u}_{+}\right) \\
& =\left.(-1)^{n} \epsilon_{u} \cdot \operatorname{sign} \frac{2 \Delta_{+}}{\phi_{-} \Delta_{-}} \frac{\partial \phi_{+}}{\partial \boldsymbol{x}}\right|_{\mathcal{F}} \boldsymbol{u}_{+}
\end{aligned}
$$

follows and this does not vanish by the cusp condition (5) since $\mathcal{F}$ is not a cusp. Thus, the last expression really defines $\epsilon_{\text {trans }}$.

Now, let $\mathcal{F}$ move along the fold curve, e.g. in the course of the pathfollowing in 2-parameter space. We will discuss how the factors of Eq. (13) change. First of all, $\Delta_{+}$and $\Delta_{-}$have fixed signs since they do not vanish by construction. Next, the sign of $\phi_{-}$flips at a \pm -bifurcation, while the sign of the derivative flips at a cusp point. Finally, $\epsilon_{u}$ flips at \pm -bifurcation points as well as at cusps points since the direction of $\boldsymbol{u}_{+}$is not inverted by continuity of the solutions of Eq. (3) while $\mathcal{P}$ is now on the other side of $\mathcal{F}$ (see Fig. 4: left to the \pm -bifurcation we have $\epsilon_{u}=1$ but $\epsilon_{u}=-1$ right to that point, analogously in the case of a cusp point). This means, $\epsilon_{\text {trans }}$ is constant along a fold curve and it is sufficient to evaluate it once when the path-following starts. 

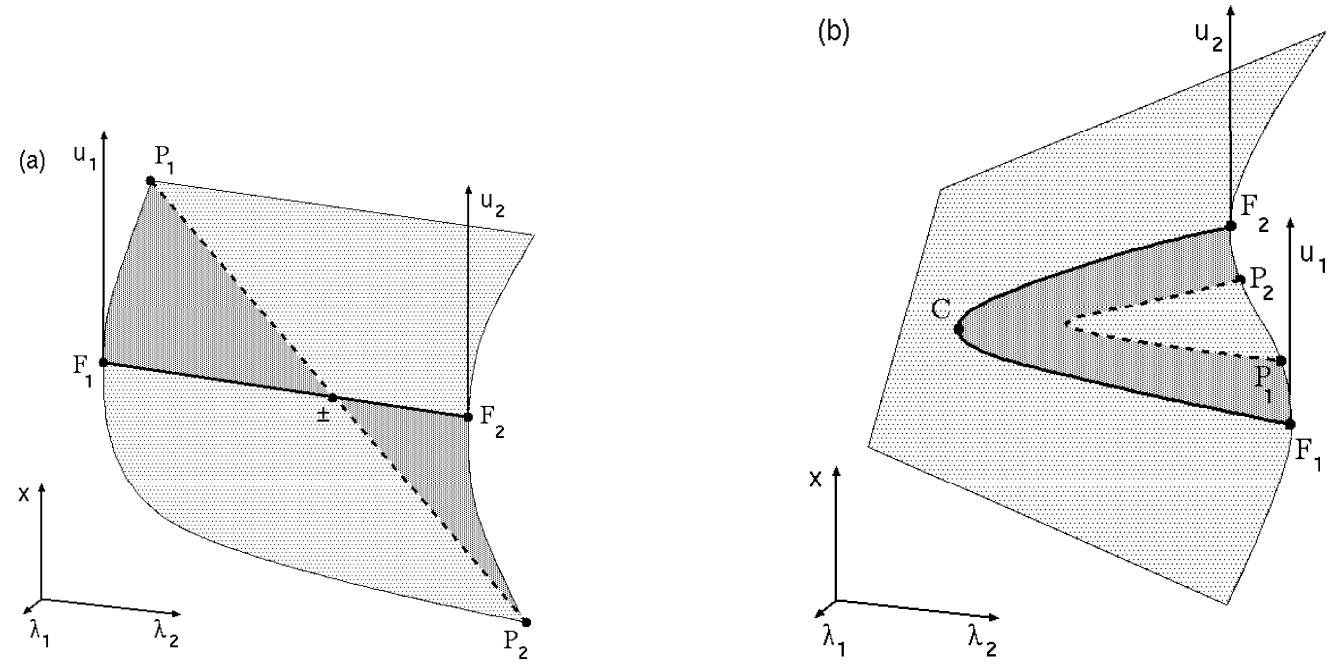

Figure 4: Change of $\epsilon_{u}$ : (a) at a \pm -bifurcation, (b) at a cusp point, the fold curves are traced from $\mathcal{F}_{1}\left(\epsilon_{u}=1\right)$ to $\mathcal{F}_{2}\left(\epsilon_{u}=-1\right)$. Solid lines: fold curves, dashed lines: flip curves, dots: bifurcation points, darkly shaded areas: transient domains.

\subsection{The inner domain}

Let $\Gamma_{2}$ be a smooth curve in the 2-parameter space that belongs to a given small neighborhood of $\mathcal{C}$ such that it joins both branches of the cusp, let its parameterization be proportional to the arc length (see Fig. 5). To be more specific, define $\Gamma_{2}$ as the intersection of the inner domain with a plane that is parallel to the tangent vector $\boldsymbol{u}_{+}$at $\mathcal{C}$. At the two endpoints of $\Gamma_{2}$ we have $\sigma=1$ and $\phi_{+}=0$ but $\sigma \neq 1$ and $\phi_{+} \neq 0$ at interior points of $\Gamma_{2}$. This means, that $\sigma(\alpha)$ and $\phi_{+}(\alpha)$ possess extrema between both endpoints. (If there are multiple extrema of $\sigma$ or $\phi_{+}$along $\Gamma_{2}$ then we consider the first from one endpoint. Later we will see that there is only one extremum each). The extrema are minima iff $\sigma(\alpha)<1$ or $\phi_{+}(\alpha)<0$. This means, that the signs of $\frac{\partial^{2} \sigma}{\partial \alpha^{2}}$ and $\frac{\partial^{2} \phi_{+}}{\partial \alpha^{2}}$ at the extreme points are inverse to the signs of $\sigma(\alpha)-1$ and $\phi_{+}(\alpha)$, respectively. Now, let $\Gamma_{2}$ tend to $\mathcal{C}$ such that it approaches smoothly the tangent to the fold curve at $\mathcal{C}$. Since this tangent is parallel to $\boldsymbol{u}_{+}$, the derivatives approximate, up to a scaling factor, $\left.\frac{\partial^{k} \sigma}{\partial \boldsymbol{x}^{k}}\right|_{\mathcal{C}}$ and $\left.\frac{\partial^{k} \phi_{+}}{\partial \boldsymbol{x}^{k}}\right|_{\mathcal{C}}$ in the $\boldsymbol{u}_{+}$ direction, respectively. We can assume that the parameterization of $\Gamma_{2}$ has 


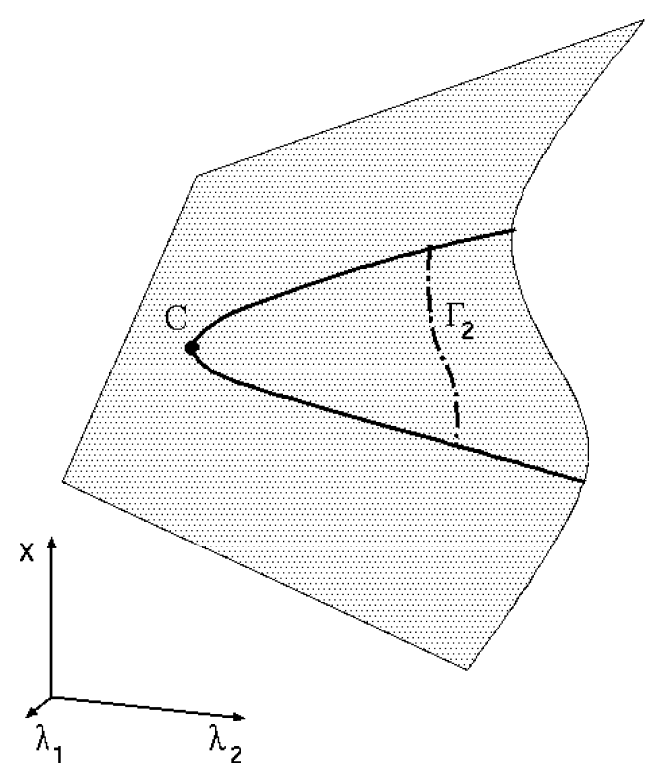

Figure 5: The curve $\Gamma_{2}$ (dash-dotted line) crossing the inner domain of the cusp point $\mathcal{C}$. Solid line: fold curve.

been chosen such a way that the scaling factor is equal to one, i.e.

$$
\begin{aligned}
\left.\frac{\partial^{k} \sigma}{\partial \alpha^{k}}\right|_{\mathcal{C}} & =\left.\frac{\partial^{k} \sigma}{\partial \boldsymbol{x}^{k}}\right|_{\mathcal{C}}\left(\boldsymbol{u}_{+}\right)^{k}, \\
\left.\frac{\partial^{k} \phi_{+}}{\partial \alpha^{k}}\right|_{\mathcal{C}} & =\left.\frac{\partial^{k} \phi_{+}}{\partial \boldsymbol{x}^{k}}\right|_{\mathcal{C}}\left(\boldsymbol{u}_{+}\right)^{k} .
\end{aligned}
$$

By Eq. (11) and the cusp condition Eq. (5), we obtain $\left.\frac{\partial \sigma}{\partial x}\right|_{\mathcal{C}} \boldsymbol{u}_{+}=0$ in the case $k=1$. This means, that the type of the reduced multiplier is essentially determined by the sign of $\left.\frac{\partial^{2} \phi_{+}}{\partial \boldsymbol{x}^{2}}\right|_{\mathcal{C}} \boldsymbol{u}_{+} \boldsymbol{u}_{+}$. Recall that this expression does not vanish for a non-degenerate cusp point. This also implies that $\sigma$ and $\phi_{+}$ really have only one extremum each since $\frac{\partial^{2} \sigma}{\partial \alpha^{2}} \neq 0$ and $\frac{\partial^{2} \phi_{+}}{\partial \alpha^{2}} \neq 0$ for cycles on $\Gamma_{2}$ if this curve is close to the tangent vector $\boldsymbol{u}_{+}$. Thus, we obtain the following equation:

$$
\epsilon_{\text {inner }}=\left.\frac{\partial^{2} \sigma}{\partial \boldsymbol{x}^{2}}\right|_{\mathcal{C}} \boldsymbol{u}_{+}=\left.(-1)^{n+1} \operatorname{sign} \frac{2 \Delta_{+}}{\phi_{-} \Delta_{-}} \frac{\partial^{2} \phi_{+}}{\partial \boldsymbol{x}^{2}}\right|_{\mathcal{C}} \boldsymbol{u}_{+} \boldsymbol{u}_{+}
$$




\subsection{The leading factor}

In this section we will analyze the factor $\frac{2 \Delta_{+}}{\phi_{-} \Delta_{-}}$occurring in Eqs. (13) and (14) in more detail. Our goal is to express it by a formula that is independent of the auxiliary vectors.

The derivative $\frac{\partial^{2} \sigma}{\partial \alpha^{2}}$ does not depend on the auxiliary vectors $\boldsymbol{l}_{ \pm}$and $\boldsymbol{r}_{ \pm}$, thus, the right hand side of Eq. (12) does not either, i.e. the dependencies on $\boldsymbol{l}_{ \pm}$and $\boldsymbol{r}_{ \pm}$cancel. For theoretical considerations, therefore, we can choose these vectors such that the expressions become as simple as possible. Let $\boldsymbol{u}$ and $\boldsymbol{v}^{T}$ be right and left zero eigenvectors of $\boldsymbol{J}-\boldsymbol{I}$ satisfying $\gamma=\boldsymbol{v}^{T} \boldsymbol{u} \neq 0$. $\left(\boldsymbol{v}^{T} \boldsymbol{u} \neq 0\right.$ holds iff +1 is a simple eigenvalue of $\boldsymbol{J}$. This will, for simplicity, be assumed.) In the following we set $\boldsymbol{r}_{ \pm}=\boldsymbol{u}, \boldsymbol{l}_{ \pm}=\boldsymbol{v}$. Then the solutions the of Eqs. (3) and (4) satisfy $\boldsymbol{u}_{ \pm}=\frac{1}{\gamma} \boldsymbol{u}$ and $\frac{1}{\gamma} \boldsymbol{v}_{ \pm}=\boldsymbol{v}$ as can be checked immediately.

Define $\Delta(\beta)=\operatorname{det}\left(\begin{array}{rr}\boldsymbol{J}-\beta \boldsymbol{I} & \boldsymbol{u} \\ \boldsymbol{v}^{T} & 0\end{array}\right)$ with $\beta \neq 1$ and $|\beta-1| \ll 1$. Let $\mu_{1}=1, \mu_{2}$, $\ldots, \mu_{n}$ be the eigenvalues of $\boldsymbol{J}$. Then $\boldsymbol{J}-\beta \boldsymbol{I}$ is nonsingular and

$$
\begin{aligned}
\Delta(\beta) & =\operatorname{det}(\boldsymbol{J}-\beta \boldsymbol{I})\left(-\boldsymbol{v}^{T^{\prime}}(\boldsymbol{J}-\beta \boldsymbol{I})^{-1} \boldsymbol{u}\right) \\
& =(1-\beta) \prod_{i=2}^{n}\left(\mu_{i}-\beta\right) \frac{-\gamma}{1-\beta} \\
& =-\gamma \prod_{i=2}^{n}\left(\mu_{i}-\beta\right)
\end{aligned}
$$

since $(\boldsymbol{J}-\beta \boldsymbol{I})^{-1} \boldsymbol{u}=\frac{1}{1-\beta} \boldsymbol{u}$. In the case of a one-dimensional map the product (and the following ones) has to be set to unity. Left and right hand side of the last equation depend continuously on $\beta$, i.e. the limit $\beta \rightarrow 1$ yields

$$
\Delta_{+}=-\gamma \prod_{i=2}^{n}\left(\mu_{i}-1\right)=(-1)^{n} \gamma \prod_{i=2}^{n}\left(1-\mu_{i}\right) .
$$

On the other hand, Eq. (10) implies $\phi_{-} \Delta_{-}=\operatorname{det}(\boldsymbol{J}+\boldsymbol{I})=2 \prod_{i=2}^{n}\left(\mu_{i}+1\right)$, i.e.

$$
(-1)^{n} \frac{2 \Delta_{+}}{\phi_{-} \Delta_{-}}=\gamma \prod_{i=2}^{n} \frac{1-\mu_{i}}{1+\mu_{i}}
$$

is, up to a factor $\epsilon_{u}$ or -1 , the leading factor of the derivatives in Eqs. (13) and (14). The sign of the product does not depend on complex eigenvalues since 
the multiplication of factors corresponding to conjugate complex eigenvalues always yields a positive number. In the case of a real eigenvalue $\mu_{i}, \frac{1-\mu_{i}}{1+\mu_{i}}$ is negative iff $\mu_{i} \notin[-1,1]$. This implies

$$
\begin{aligned}
\operatorname{sign}(-1)^{n} \frac{2 \Delta_{+}}{\phi_{-} \Delta_{-}} & =(-1)^{\#\left\{i: \mu_{i} \notin[-1,1]\right\}} \operatorname{sign} \gamma \\
& =(-1)^{\#\left\{i:\left|\mu_{i}\right|>1\right\}} \operatorname{sign} \gamma .
\end{aligned}
$$

The last equation follows from the fact that one can include arbitrarily many pairs of conjugate complex eigenvalues into the product.

Let $\epsilon_{u}$ correspond to the direction of $\boldsymbol{u}$. Together with Eqs. (13) and (6) we obtain

$$
\begin{aligned}
\epsilon_{\text {trans }} & =\left.\epsilon_{u} \cdot(-1)^{\#\left\{i:\left|\mu_{i}\right|>1\right\}} \cdot \operatorname{sign} \gamma \cdot \operatorname{sign} \frac{\partial \phi_{+}}{\partial \boldsymbol{x}}\right|_{\mathcal{F}} \boldsymbol{u} \\
& =-\left.\epsilon_{u} \cdot(-1)^{\#\left\{i:\left|\mu_{i}\right|>1\right\}} \cdot \operatorname{sign} \frac{1}{\gamma} \boldsymbol{v}^{T} \frac{\partial \boldsymbol{J}}{\partial \boldsymbol{x}}\right|_{\mathcal{F}} \boldsymbol{u} \boldsymbol{u} .
\end{aligned}
$$

Analogously, Eq. (14) yields

$$
\epsilon_{\text {inner }}=-\left.(-1)^{\#\left\{i:\left|\mu_{i}\right|>1\right\}} \cdot \operatorname{sign} \gamma \frac{\partial^{2} \phi_{+}}{\partial \boldsymbol{x}^{2}}\right|_{\mathcal{C}} \boldsymbol{u} \boldsymbol{u}
$$

where the derivative is given by Eq. (7). These expressions do not change if the eigenvectors are rescaled by any factors. In particular, one can replace $\boldsymbol{u}$, $\boldsymbol{v}$ by the solutions $\boldsymbol{u}_{+}$and $\boldsymbol{v}_{+}$of Eqs. (3) and (4), respectively, with arbitrary auxiliary vectors.

\subsection{Low dimensional maps}

In the case of a one-dimensional map we can always set $\boldsymbol{r}_{+}=\boldsymbol{l}_{+}=\boldsymbol{r}_{-}=$ $\boldsymbol{l}_{-}=1$ in Eq. (3) yielding $\Delta_{+}=\Delta_{-}=-1, \boldsymbol{u}_{+}=\boldsymbol{v}_{+}=1, \phi_{+}=-\mu_{1}+1$, $\phi_{-}=-\mu_{1}-1$, and $\sigma=-\frac{\theta}{\eta}=\frac{\mu_{1}}{1}$ where $\mu_{1}$ is the single eigenvalue of $\boldsymbol{J}$. (The last equation caused the term "reduced" multiplier for $\sigma$ in the case of higher dimensional maps.) The eigenvalue $\mu_{1}$ varies between a fold and a flip curve from +1 to -1 and so does $\sigma$, i.e. $\epsilon_{\text {trans }}=1$.

A similar situation holds for dissipative two-dimensional maps, i.e. maps with the property $|\operatorname{det} \boldsymbol{J}(\boldsymbol{x})|=\left|\mu_{1} \mu_{2}\right|<1$. The denominator of $\sigma=\frac{\mu_{1}+\mu_{2}}{1+\mu_{1} \mu_{2}}$ 
does not vanish, thus the numerator changes its sign when a cycle is moved from the fold curve to the flip curve implying $\epsilon_{\text {trans }}=1$. Moreover, $\left|\mu_{2}\right|<1$ holds for fold points since $\mu_{1}=1$.

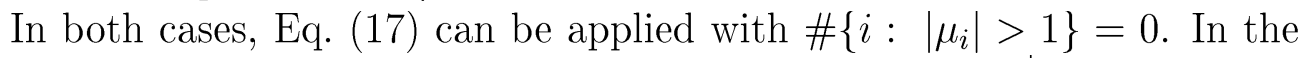
case $n=1$, the derivative in Eq. (17) can be replaced by $\left.\frac{\partial^{3} f}{\partial x^{3}}\right|_{\mathcal{C}}>0$ since the matrix $\boldsymbol{A}$ in Eq. (7) vanishes.

Proposition 3.1 In the case of a one-dimensional or a dissipative twodimensional map the communication area is a SAA iff $\epsilon_{\text {inner }}=1$. In the case of a one-dimensional map this is equivalent to $\left.\frac{\partial^{3} f}{\partial x^{3}}\right|_{\mathcal{C}}>0$. while for dissipative two-dimensional maps this is equivalent to $\left.\boldsymbol{v}^{T} \boldsymbol{u} \cdot \frac{\partial^{2} \phi_{+}}{\partial \boldsymbol{x}^{2}}\right|_{\mathcal{C}} \boldsymbol{u u}>0$ where $\boldsymbol{u}$ and $\boldsymbol{v}^{T}$ are the right and left eigenvectors of $\boldsymbol{J}$.

\subsection{The algorithm}

The type of a communication area is determined by multiplication of $\epsilon_{\text {trans }}$ by $\epsilon_{\text {inner }}$ given by Eqs. (16) and (17). The factors cancel partially. More precisely, $\operatorname{sign} \gamma=\operatorname{sign} \boldsymbol{v}_{+}^{T} \boldsymbol{u}_{+}$changes on the path from $\mathcal{F}$ to $\mathcal{C}$ iff a second eigenvalue passes +1 while $(-1)^{\#\left\{i:\left|\mu_{i}\right|>1\right\}}$ changes iff a real eigenvalue passes \pm 1 . This means, the factors $(-1)^{\#\left\{i:\left|\mu_{i}\right|>1\right\}} \cdot \operatorname{sign} \gamma$ cancel up to the factor $(-1)^{b}$ where $b$ is the number of \pm -bifurcations between $\mathcal{F}$ and $\mathcal{C}$.

Proposition 3.2 Assume that $\mathcal{F}$ is a point, but neither a \pm -bifurcation nor a cusp point, on the same fold curve as the cusp point $\mathcal{C}$ and that $b \pm$ bifurcation points are between both points. Then the communication area at $\mathcal{C}$ is a $S A A$ iff

$$
\left.\left.(-1)^{b} \cdot \epsilon_{u}(\mathcal{F}) \cdot \operatorname{sign} \frac{\partial \phi_{+}}{\partial \boldsymbol{x}}\right|_{\mathcal{F}} \boldsymbol{u}_{+} \cdot \operatorname{sign} \frac{\partial^{2} \phi_{+}}{\partial \boldsymbol{x}^{2}}\right|_{\mathcal{C}} \boldsymbol{u}_{+} \boldsymbol{u}_{+}<0
$$

The proposition suggests the following algorithm.

1. The usual way of the numerical bifurcation analysis consists of the following steps:

(a) a first cycle $\boldsymbol{x}$ is determined for fixed parameters 
(b) path-following of this cycle with respect to one variable parameter and fold and flip points on the curve have to be detected and computed

(c) the fold points of step $1 \mathrm{~b}$ become the object of path-following when two parameters are varied and, among others, cusp points have to be detected and computed

2. If in step $1 \mathrm{~b}$ a fold point $\mathcal{F}$ has been computed then $\left.\frac{\partial \phi_{+}}{\partial \boldsymbol{x}}\right|_{\mathcal{F}}$ is the vector composed of expressions in Eq. (6) where $\alpha$ runs through the components of $\boldsymbol{x}$. It is a row of the Jacobian matrix needed in Newton's method for computing the fold point, and $\left.\frac{\partial \phi_{+}}{\partial \boldsymbol{x}}\right|_{\mathcal{F}} \boldsymbol{u}_{+}$is simply its scalar product with $\boldsymbol{u}_{+}$.

3. The path is followed from $\mathcal{F}$ to the next flip point $\mathcal{P}$. $\epsilon_{u}$ is the sign of the scalar product of $\boldsymbol{u}_{+}$with the tangent vector to the curve at $\mathcal{F}$ used in the path-following and multiplied by -1 if $\mathcal{P}$ lies before $\mathcal{F}$ on the path. The value $\epsilon_{\text {trans }}=\left.\epsilon_{u} \cdot \frac{\partial \phi_{+}}{\partial \boldsymbol{x}}\right|_{\mathcal{F}} \boldsymbol{u}_{+}$is assigned to the point $\mathcal{F}$.

4. If in step 1c a cusp point $\mathcal{C}$ has been computed then $\epsilon_{\text {inner }}=$ $\left.\frac{\partial^{2} \phi_{+}}{\partial \boldsymbol{x}^{2}}\right|_{\mathcal{C}} \boldsymbol{u}_{+} \boldsymbol{u}_{+}$is obtained similarly to $\left.\frac{\partial \phi_{+}}{\partial \boldsymbol{x}}\right|_{\mathcal{F}} \boldsymbol{u}_{+}$in step 2 (recall that $\left.\frac{\partial^{2} \phi_{+}}{\partial \boldsymbol{x}^{2}}\right|_{\mathcal{C}} \boldsymbol{u}_{+}$equals the derivative of the function $\phi_{2}$ in the cusp condition Eq. (5)).

5. Counting the number of \pm -bifurcations between $\mathcal{F}$ and $\mathcal{C}$ in step $1 \mathrm{c}$ gives $b$.

6. The left hand side of Eq. (18) is evaluated.

It should be emphasized that additionally to the usual method computing a cusp point only a few scalar products of known vectors have to be calculated and the \pm -bifurcations have to be counted. Steps 2, 3, 5, and 6 can be discarded if the map satisfies the conditions of Proposition 3.1. Step 3 is a bit unsafe: it can happen that no flip point can be found within an acceptable path-length. But as is our experience, in most cases the flip point is very close to $\mathcal{F}$. 


\subsection{Area transitions}

When performing path-following of the cusp point by variation of a third parameter, area transitions can occur. By Proposition 3.2, the area transition is accompanied with the change of the sign of $\frac{\partial^{2} \phi_{+}}{\partial x^{2}} \boldsymbol{u}_{+} \boldsymbol{u}_{+}$or of an odd change of the number of \pm -bifurcations between $\mathcal{F}$ and $\mathcal{C}$. Since $\mathcal{F}$ is a fixed cycle that is not a \pm -bifurcation point the last situation can occur only if a \pm bifurcation point passes $\mathcal{C}$. With other words, an area transition takes place if the cusp becomes degenerate or if one eigenvalue $\mu_{i}$ passes the value -1 . Comparing this observation with the considerations of Sec. 2.1, the following is proved.

Proposition 3.3 A SAA↔CRA transition occurs iff the cusp point becomes degenerate while a $S A A \leftrightarrow S P A$ transition occurs iff a \pm -bifurcation passes the cusp point.

The equation system for the point in the state-parameter space where an area transition occurs is simply the equation system for the cusp point extended by one further equation that describes the additional condition:

$$
\begin{array}{r}
\boldsymbol{f}\left(\boldsymbol{x}, \lambda_{1}, \lambda_{2}, \lambda_{3}\right)-\boldsymbol{x}=\boldsymbol{0} \\
\phi_{+}\left(\boldsymbol{x}, \lambda_{1}, \lambda_{2}, \lambda_{3}\right)=0 \\
\phi_{c}\left(\boldsymbol{x}, \lambda_{1}, \lambda_{2}, \lambda_{3}\right)=0 \\
\phi_{a}\left(\boldsymbol{x}, \lambda_{1}, \lambda_{2}, \lambda_{3}\right)=0
\end{array}
$$

where $\phi_{+}$is given by Eq. (3) with $\boldsymbol{M}_{+}=\boldsymbol{J}-\boldsymbol{I}, \phi_{c}$ is given by the cusp condition (5), and the additional function $\phi_{a}$ is given by Eq. (8) in case of a SAA $\leftrightarrow$ CRA transition, or by Eq. (3) with $\boldsymbol{M}_{a}=\boldsymbol{J}+\boldsymbol{I}$ in case of a SAA $\leftrightarrow$ SPA transition. The Jacobian of Eq. (19) evaluated at the solution point is not singular iff the solution is a co-dimension-3 bifurcation, the generic situation. In this case, this equation system can be solved by Newton's method.

In 3-parameter space, there exists a curve of \pm -bifurcations points which intersects the curve of cusp points at the point of the SAA $\leftrightarrow$ SPA transition. This curve can be obtained by path-following since one point is known, the point of the area transition, and the tangent $\left(\boldsymbol{t}_{x}, t_{1}, t_{2}, t_{3}\right)$ of the curve at this point can easily be computed as will be shown next. The matrix of the linear equation system below is that used in Newton's method to compute 
the point of the area transition and, therefore, it is nonsingular.

$$
\left(\begin{array}{cccc}
\boldsymbol{J}-\boldsymbol{I} & \frac{\partial f}{\partial \lambda_{1}} & \frac{\partial \boldsymbol{f}}{\partial \lambda_{2}} & \frac{\partial f}{\partial \lambda_{3}} \\
\frac{\partial \phi_{+}}{\partial \boldsymbol{x}} & \frac{\partial \phi_{+}}{\partial \lambda_{1}} & \frac{\partial \phi_{+}}{\partial \lambda_{2}} & \frac{\partial \phi_{+}}{\partial \lambda_{3}} \\
\frac{\partial \phi_{c}}{\partial \boldsymbol{x}} & \frac{\partial \phi_{c}}{\partial \lambda_{1}} & \frac{\partial \phi_{c}}{\partial \lambda_{2}} & \frac{\partial \phi_{c}}{\partial \lambda_{3}} \\
\frac{\partial \phi_{a}}{\partial \boldsymbol{x}} & \frac{\partial \phi_{a}}{\partial \lambda_{1}} & \frac{\partial \phi_{a}}{\partial \lambda_{2}} & \frac{\partial \phi_{a}}{\partial \lambda_{3}}
\end{array}\right)\left(\begin{array}{c}
\boldsymbol{t}_{x} \\
t_{1} \\
t_{2} \\
t_{3}
\end{array}\right)=\left(\begin{array}{l}
\boldsymbol{O} \\
0 \\
1 \\
0
\end{array}\right)
$$

Canceling the third equation results in the homogeneous equation system for the tangent to be calculated (it includes the derivatives of the functions defining the bifurcation with respect to all variables). Thus, the nonsingular inhomogeneous equation system yields an unnormalized tangent. It should be mentioned, that this procedure does not work in the case of a degenerate cusp point since cancelation of the equation $\phi_{c}=0$ yields an equation system that does not describe a bifurcation situation.

\section{Comparison with Carcassès' Method}

\subsection{Carcassès' method}

Before the comparison can be done, Carcassès' method [2] is to be described shortly. This method to solve the problems

(A) determination of the type of a communication area

(B) computation of the points in the state-parameter space where an area transition occurs

is based on the solution of equation systems of the form

$$
\begin{aligned}
\boldsymbol{f}(\boldsymbol{x}, \lambda)-\boldsymbol{x} & =\boldsymbol{0} \\
\psi_{1}(\boldsymbol{x}, \lambda) & =0 \\
\psi_{2}(\boldsymbol{x}, \lambda) & =0
\end{aligned}
$$

with $\lambda=\left(\lambda_{1}, \lambda_{2}\right)$ or $\lambda=\left(\lambda_{1}, \lambda_{2}, \lambda_{3}\right)$ in problems (A) and (B), respectively. The functions $\psi_{1,2}$ are determinants like

$$
\psi_{1}=\operatorname{det}\left(\begin{array}{cc}
\boldsymbol{J}-\boldsymbol{I} & \frac{\partial \boldsymbol{f}}{\partial \lambda_{2}} \\
\eta \frac{\partial \theta}{\partial \boldsymbol{x}}-\theta \frac{\partial \eta}{\partial \boldsymbol{x}} & \eta \frac{\partial \theta}{\partial \lambda_{2}}-\theta \frac{\partial \eta}{\partial \lambda_{2}}
\end{array}\right)
$$


where $\eta$ and $\theta$ are defined in Sec. 3.1. The solutions of Eq. (21) are called parametric singularities, among them are the cusp points.

Problem (A) is solved as follows. Path-following of Eq. (21) is performed, where one of the equations $\psi_{1,2}(\boldsymbol{x}, \lambda)=0$ is discarded, and points satisfying the full equation system have to be detected and computed. If such a point has been encountered then two specific expressions (called $b_{1}$ and $b_{2}$ ) and $\sigma=-\frac{\theta}{\eta}$ have to be evaluated. These values determine the type of the parametric singularity and, as a consequence, the type of the communication area.

Problem (B) is solved by path-following of Eq. (21) with respect to a third parameter. The points, where $\sigma= \pm 1$ or $b_{2}=0$ holds, have to be detected and calculated.

\section{$4.2 \quad$ Numerical complexity}

The numerical complexity of both methods is determined by the following facts.

1. Since $\eta$ and $\theta$ depend on the first derivatives of $\boldsymbol{f}(\boldsymbol{x}, \lambda)$, the determinants and eventually Eq. (21) depend on the second derivatives. Furthermore, $b_{2}$ is an expression composed of the derivatives of many determinants of form (22), i.e. $b_{2}$ depends on the third derivatives. By contrast, in our method only the equation system for the cusp points depend on the second (non-degenerate cusps) or third derivatives (degenerate cusps). Of course, the use of Newton's method causes in each case that derivatives of one higher order are needed.

2. Computing determinants like (22) is equivalent to solving a linear equation system with the same matrix (provided that the matrix entries are known). But the computation of the derivatives of the determinants (22) (e.g. in Newton's method or to calculate $b_{2}$ ) is much more complex then evaluating Eqs. (6) or (7).

3. The determination of the coefficients of the characteristic polynomial needs $\sim n^{3}$ operations, i.e. it is as complex as the solution of a linear equation system with the same matrix. Calculating the derivatives (recall that $b_{2}$ depends on the second derivative of $\eta$ and $\theta$ ) is then 
as complex as calculating the derivatives of the determinants. Our method does not need the characteristic polynomial at all.

4. Information gained from the path-following of curves of the several bifurcations are not utilized in Carcassès' method. As already mentioned, our method strongly utilized such information.

\subsection{Other differences}

Another disadvantage of Carcassès' method is that it implicitly assumes that the reduced multiplier $\sigma$ ranges from -1 to +1 between fold and flip curves. If this assumption is not satisfied then similarly to our method specific signs have to be inverted. But the assumption is never checked, this means that possibly the type of a communication area will be determined incorrectly. That this situation can really occur is shown in Sec. 5.3. The assumption is satisfied for one-dimensional and dissipative two-dimensional maps (see Sec. 3.5). This is the reason why the method worked correctly in the examples studied in [1] and [13].

Since Carcassès' method is based on the characteristic polynomial $\chi(\mu)$ (via the reduced multiplier $\sigma$ ) it is applicable to finite-dimensional maps only. Our method utilizes only such information that can be gained also in the case of infinite-dimensional maps (defined, e.g., in a Banach space), i.e. the method should be applicable to such maps. But this enforces that the foundation of the method has to be revisited.

Nevertheless, Carcassès' method has also advantages compared to our method. The main advantage is that also SPA and CRA can be distinguished and that SPA $\leftrightarrow$ CRA transitions can be determined. Furthermore, the method can detect bifurcation situations which are beyond the scope of communication areas in the sense of Sec. 1, e.g. whether a fold or flip curve is closed. These capabilities are caused by the fact that the method works in some sense globally. By contrast, our method works locally. A consequence is that it is impossible to decide whether the cusp (when it has been found and examined) belongs really to a communication area, i.e. whether the other curves constituting the communication area do exist. On the other hand, this means, that the change of the sign of Eq. (18) may signal also other global changes in the bifurcation diagram. 


\begin{tabular}{r|rr|rrrr} 
no. & sign of (18) & type & $x$ & $y$ & $a$ & $c$ \\
\hline 1 & + & SPA & -1.675490 & -0.427148 & -1.812033 & -0.413519 \\
2 & + & SPA & -2.137116 & -0.200239 & 1.761686 & -0.348546 \\
3 & - & SAA & -2.231921 & -0.825089 & 1.864383 & -1.945378 \\
4 & + & SPA & -1.912922 & -0.530341 & -0.251560 & 0.773379 \\
5 & - & SAA & -1.806427 & -0.282897 & -3.819128 & -3.440879 \\
6 & + & SPA & -2.286317 & 0.282957 & 1.811106 & -3.730972 \\
7 & - & SAA & -1.139927 & 0.550181 & -2.342272 & 2.038960 \\
8 & + & SPA & -0.707295 & 0.789517 & -3.867172 & 0.596430
\end{tabular}

Table 1: The communication areas in the $(a, c)$-parameter space and the coordinates of their cusp points.

\section{Example}

\subsection{Communication areas}

The new method is to be demonstrated by an example. We have considered the same map as Carcassès [1]:

$$
\boldsymbol{f}(\boldsymbol{x}, \lambda)=\left(\begin{array}{c}
c+(1+a) x+b x^{2}+x^{4}+y \\
d x
\end{array}\right)
$$

with $\boldsymbol{x}^{T}=(x, y)$ and $\lambda=(a, b, c, d)$; cycles of period 3 have been studied. The parameters were set initially to $(a, b, c, d)=(-1.0,-3.0,-0.2,0.4)$. Starting the path-following in the $a$-parameter space at $(x, y, a)=$ $(-1.763087,-0.624706,-1.0), 7$ fold and 7 flip points have been found (fold and flip points are grouped in pairs). Then path-following in the $(a, c)$ parameter space has been performed starting at these bifurcation points. The seven curve pairs join together to two pairs of curves: the curves of one pair are closed and its fold curve contains two cusp points (no. 1 and 2), the curves of the other pair are not closed and the fold curve contains six cusp points (no. 3 through 8). The results are shown in Table 1 and Fig. 6, the types of the communication areas have been determined by visual inspection of the figure. 

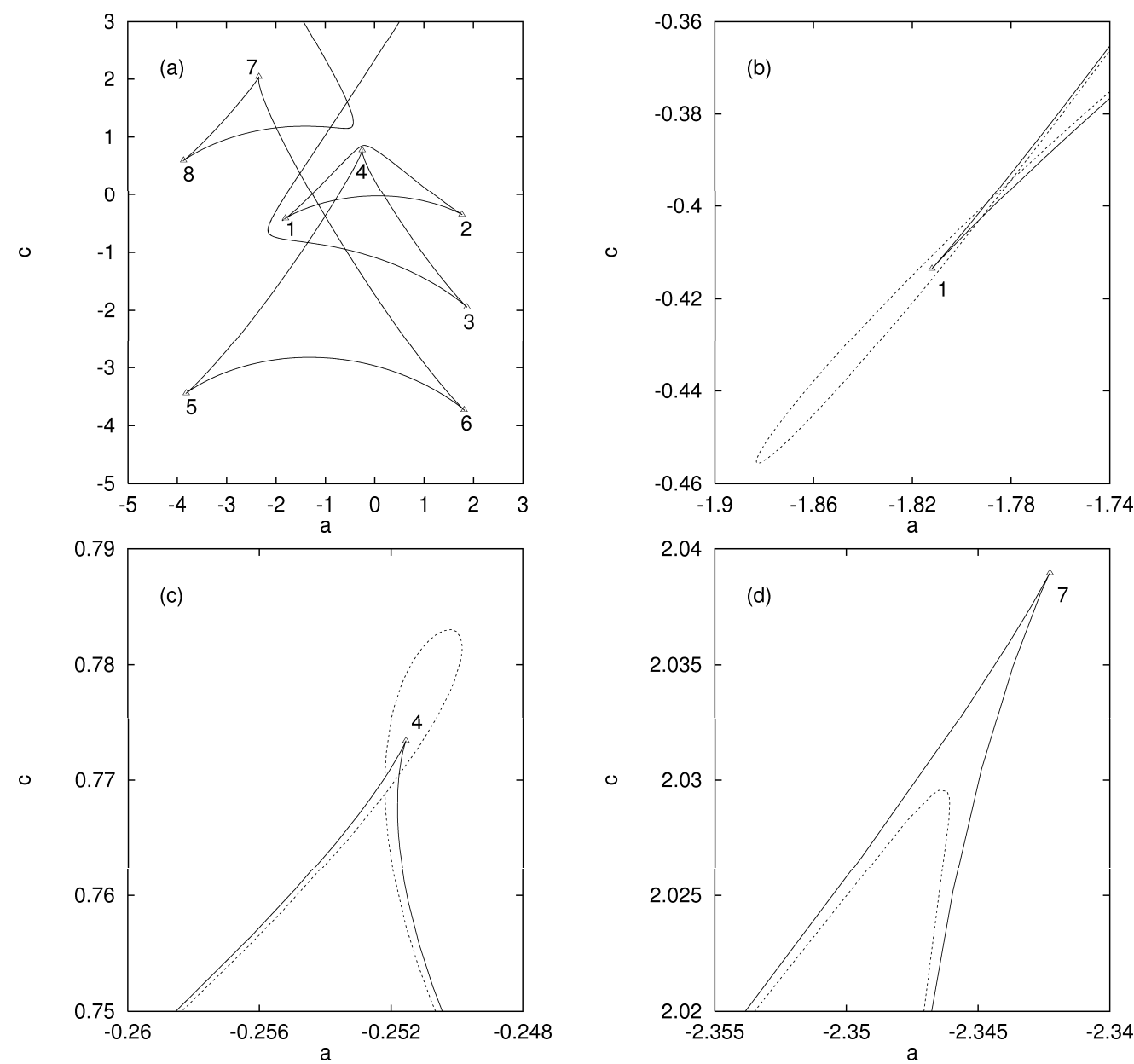

Figure 6: Bifurcation diagrams in the $(a, c)$-parameter space: (a) synopsis of fold curves, flip curves, and cusp points, (b)-(d) enlargements of (a) showing two spring areas, (cusp 1 and cusp 4), as well as one saddle area, (cusp 7). Solid lines: fold curves, dashed lines: flip curves, triangles: cusp points. 


\begin{tabular}{r|cc|rrrrr} 
no. & sign of (18) & type & $x$ & $y$ & $a$ & $b$ & $c$ \\
\hline 3 & - & SAA & -1.571348 & -0.440542 & -0.015028 & -1.172028 & -0.730 \\
3 & + & CRA & -1.566199 & -0.411577 & -0.010548 & -1.122764 & -0.715 \\
3 & + & CRA & -1.589890 & -0.411532 & -0.032288 & -1.213020 & -0.700
\end{tabular}

Table 2: The communication areas in the $(a, b)$-parameter space on both sides of the SAA $\leftrightarrow$ CRA transition.

\subsection{Area transitions}

The path-following of the cusp points in the $(a, b, c)$-parameter has shown that the curve corresponding to cusp 3 contains a degenerate cusp point near $(x, y, a, b, c)=(-1.5605,-0.4159,-0.0074,-1.1102,-0.7154)$. On both sides of this point and near it, at $c=-0.730, c=-0.715$, and $c=-0.700$, bifurcation diagrams in the $(a, b)$-parameter space have been computed. The results are shown in Table 2 and Fig. 7 . It can be seen that between $c=-0.730$ and $c=-0.700$ a SAA $\leftrightarrow$ CRA transition occurs.

The determinant of the Jacobian of $(23)$ is equal to $-d$ independently of $(x, y)$, i.e. that of the 3 -periodic cycles is equal to $-d^{3}$. This means, that path-following of a fold point or a cusp point (i.e. one eigenvalue is equal to one) by variation of $d$ encounters at $d=1$ a \pm bifurcation. Thus, cusp 1 has been path-followed in the $(a, c, d)$-parameter space. As expected, a \pm -bifurcation point has been found at $(x, y, a, c, d)=$ $(-1.859032,-1.150808,-0.491048,0.733427,1.000000)$. On both sides of this point, at $c=0.7$ and $c=0.8$ bifurcation diagrams in the $(a, d)$-parameter space have been computed. The results are shown in Fig. 8. It can be seen that between $c=0.7$ and $c=0.8$ a SAA $\leftrightarrow \mathrm{SPA}$ transition occurs. Moreover, the fold curve crosses for $c=0.8$ the value $d=1$ where \pm -bifurcations occur.

\subsection{An odd transient domain}

It remains to show that the value of $\epsilon_{\text {trans }}$ is not trivial, i.e. that it can take on the value -1 . To this end we have extended the map (23):

$$
\boldsymbol{f}(\boldsymbol{x}, \lambda)=\left(\begin{array}{c}
c+(1+a) x+b x^{2}+x^{4}+y \\
d x \\
2 z
\end{array}\right)
$$




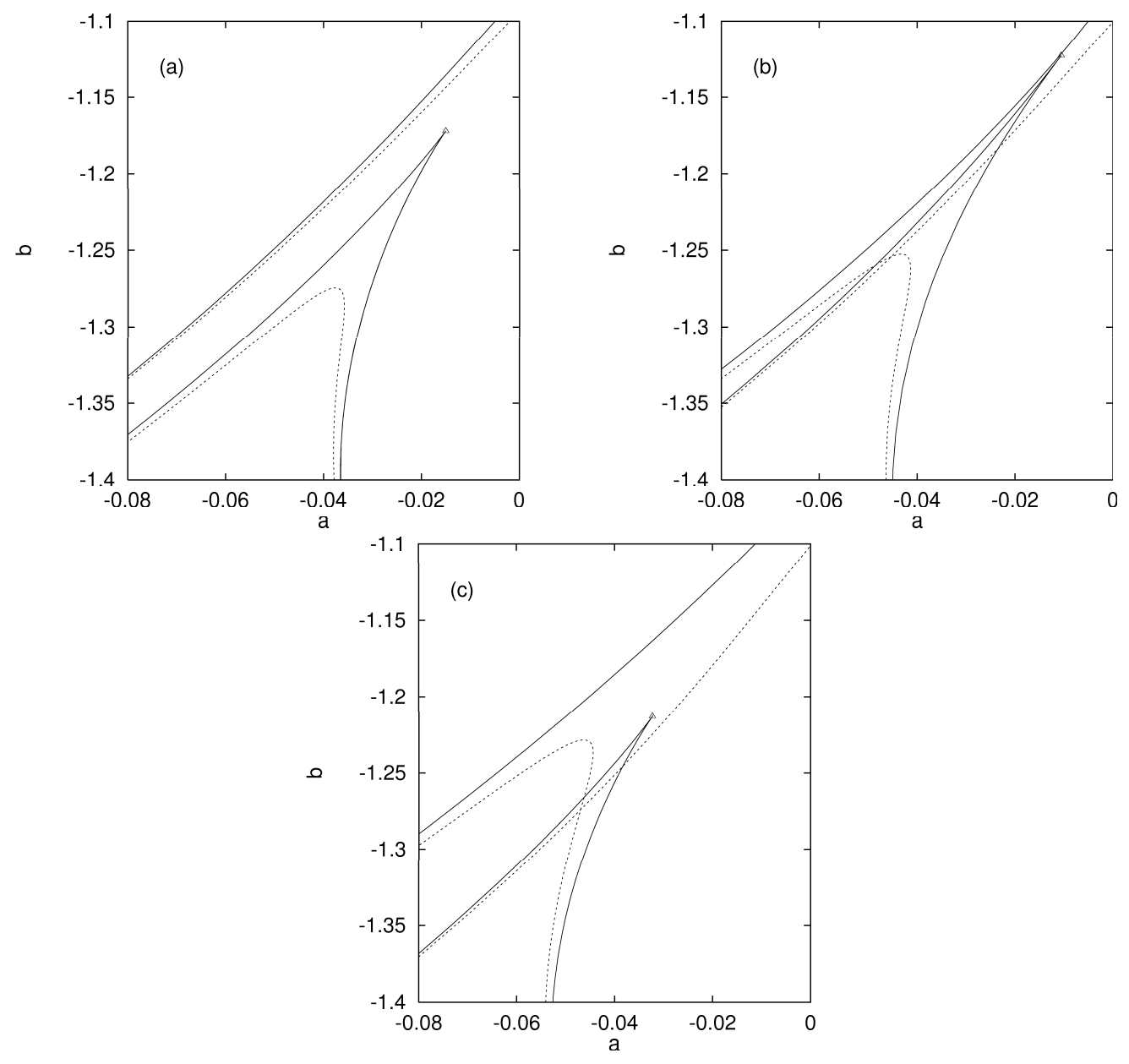

Figure 7: Three stages of the SAA $\leftrightarrow$ CRA transition at cusp 3: (a) the SAA at $c=-0.730,(\mathrm{~b})$ near the transition at $c=-0.715$, (c) the CRA at $c=-0.700$. Solid lines: fold curves, dashed lines: flip curves, triangles: cusp points. 


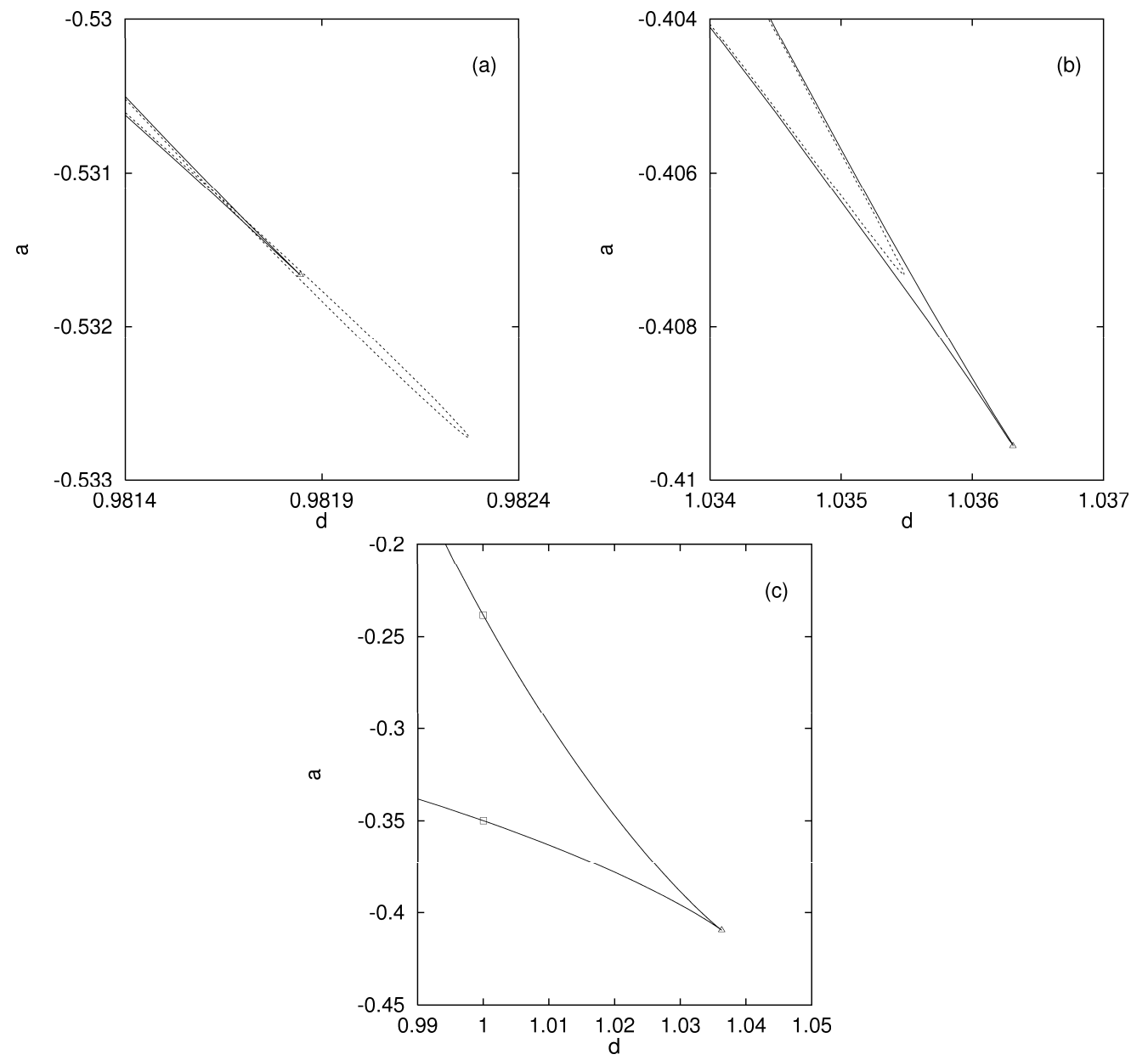

Figure 8: Two stages of the SAA $\leftrightarrow \mathrm{SPA}$ transition at cusp 1: (a) the SAA at $c=0.7$, (b) and (c) the SPA at $c=0.8$. Solid lines: fold curves, dashed lines: flip curves, triangles: cusp points, squares \pm -bifurcation points. 

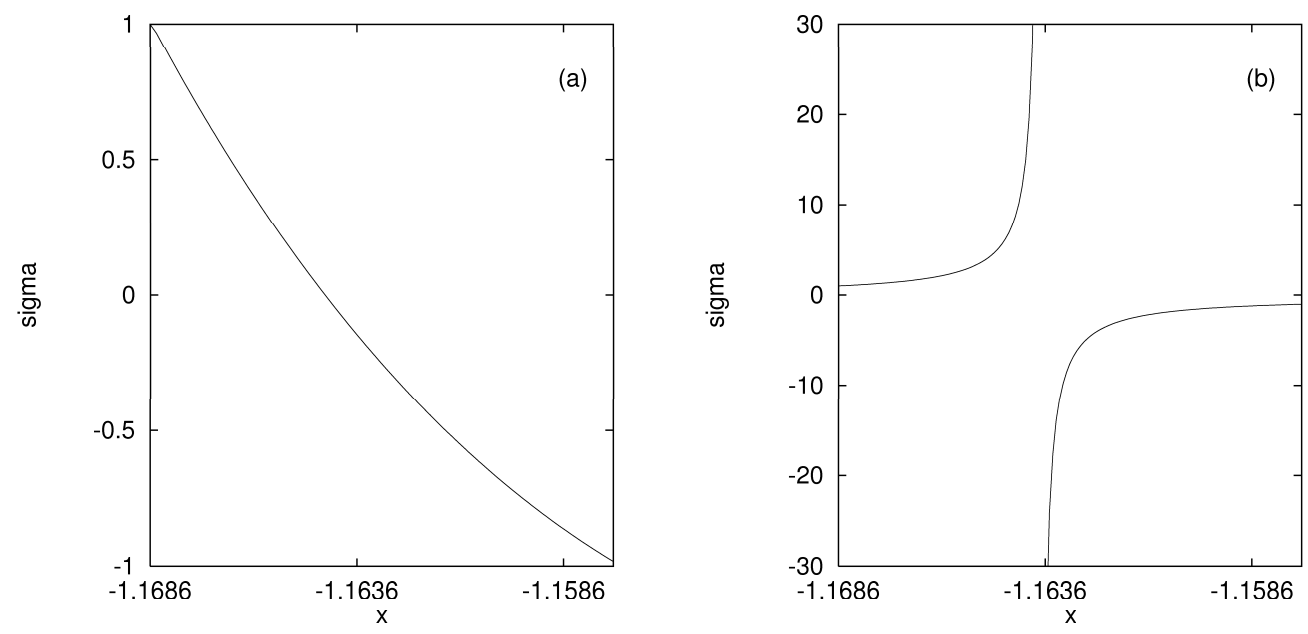

Figure 9: The graphs of $\sigma$ : (a) the original map Eq. (23), (b) the extended map Eq. (24).

with $\boldsymbol{x}^{T}=(x, y, z)$. Since the additional variable $z$ is completely decoupled from the others and does not depend on parameters, the bifurcation structure is essentially the same as for the original map (23). In particular, the same communication areas occur at the same parameter values. One difference is the stability of the cycles: all cycles are unstable because of the eigenvalue $\mu_{3}=2$. Another difference is that $\mu_{3}$ causes one additional factor -1 in Eq. (16), i.e. now we have $\epsilon_{\text {trans }}=-1$ and $\sigma$ passes through $\infty$ when a transient domain is crossed along a curve $\Gamma_{1}$.

The reduced multiplier $\sigma$ has been computed for both maps in the $(a, c)$ parameter space along the line $c=2.028=$ const from the fold to the flip curve near cusp 7 (this corresponds to a straight horizontal line in Fig. 6d). The graphs of $\sigma$ versus the variable $x$ are shown in Fig. 9 (the curve can be parameterized by $x$ ). As expected, $\sigma$ passes $\infty$ in case of the map (24).

\subsection{Technical remarks}

The computations have been performed by the program package CANDYS/QA [4], but the program has been essentially improved since that time [8]. The program computes the bifurcation points of all types mentioned in this paper, with the exception of degenerate cusp points which can be de- 
tected only. The bifurcation points are computed by the equation systems described in Sec. 2.2. At the computed bifurcation points short stubs of all branching off curves are determined, thus, it is easy to start the pathfollowing anew.

Since our example map Eq. (23) is known to be dissipative, simply Eq. (16) has been evaluated to determine the signs listed in Tables 1 and 2. The full procedure, i.e. including the determination of $\epsilon_{\text {trans }}$, has not yet been implemented in CANDYS/QA.

\section{Conclusions}

In this paper we have presented a new method to determine the type of a communication area by numerical means. It reduces the numerical effort to the calculation of a few scalar products when the communication area's cusp point has been computed by a standard algorithm. This method is an alternative to that proposed by Carcassès [2]. The disadvantage of our method is that spring areas and crossroad areas cannot be distinguished as Carcassès' method can. Closing this gap will be the topic of future work.

The method works essentially locally. A consequence is, that it is impossible to decide whether the cusp, when it has been found and examined, belongs to a communication area, i.e. whether the other curves constituting the communication area do exist.

The paper also shows that saddle area $\leftrightarrow$ spring area transitions can exist which have not yet been considered in the literature. These transitions are accompanied with the passage of a \pm -bifurcation through the communication area's cusp point. As for the \pm -bifurcations, saddle area $\leftrightarrow$ spring area transitions can occur only if the map is sufficiently complex; they do not occur in one-dimensional and dissipative two-dimensional maps. Finally, analytical conditions for the occurrence of the area transitions considered here have been offered. 


\section{References}

[1] Carcassès, J. P.: Determination of different configurations of fold and flip bifurcation curves of a one or two-dimensional map. Int. J. Bifurcation \& Chaos 3 (1993), 869-902.

[2] Carcassès, J. P.: Singularities of the parameter plane of an $n$ dimensional map. Determination of different configurations of fold and flip bifurcation curves. Int. J. Bifurcation \& Chaos 5 (1995), 419-447.

[3] Carcassès, J. P., Mira, C., Bosch, M., Simó, C., and TatJER, J. C.: Crossroad area-spring area transition, (i) parameter plane representation. Int. J. Bifurcation \& Chaos 1 (1991), 183-196.

[4] Feudel, U., And Jansen, W.: CANDYS/QA - a software system for the qualitative analysis of nonlinear dynamical systems. Int. J. Bifurcation \& Chaos 2 (1992), 773-794.

[5] Govaerts, W .: Computation of Takens-Bogdanov type bifurcations with arbitrary codimension. SIAM J. Numer. Anal. 30 (1993), 11211133.

[6] Griewank, A., And Reddien, G. W.: Characterization and computation of generalized turning points. SIAM J. Numer. Anal. 21 (1984), $176-186$.

[7] Griewank, A., And Reddien, G. W.: Computation of cusp singularities for operator equations and their discretizations. J. Comp. Appl. Maths. 26 (1989), 133-153.

[8] Jansen, W.: CANDYS/QA: Algorithms, programs, and user's manual. Tech. Rep. NLD-027, Interdisziplinäres Zentrum für nichtlineare Dynamik, Universität Potsdam, 1995.

[9] Jepson, A. D., And Spence, A.: On a reduction process for nonlinear equations. SIAM J. Math. Anal. 20 (1989), 39-56.

[10] MirA, C.: Chaotic Dynamics. From the One-Dimensional Endomorphism to the Two-Dimensional Diffeomorphism. World Scientific, Singapore, 1987. 
[11] Mira, C., AND CARCAssès: On the crossroad area-saddle area and crossroad area-spring area transitions. Int. J. Bifurcation \& Chaos 1 (1991), 641-665.

[12] Mira, C., Carcassès, J. P., Bosch, M., Simó, C., and Tatjer, J. C.: Crossroad area-spring area transition, (ii) foliated parametric representation. Int. J. Bifurcation $\&$ Chaos 1 (1991), 309-348.

[13] Mira, C., AND QRiouet, M.: On a "crossroad area-spring area" transition occuring in a Duffing-Raleigh equation with a periodical excitation. Int. J. Bifurcation \& Chaos 3 (1993), 1029-1037. 